\title{
Growth, Characterization and NLO activity of Fe (III) doped Ni L-Histidine Hydrochloride Monohydrate Crystals
}

\author{
V.Parvathi, J. Sai Chandra and Y.Sunandamma \\ Department of Chemistry, Acharya Nagarjuna University, Nagarjuna Nagar- 522510,A.P, INDIA
}

\begin{abstract}
Fe}(\mathrm{III})$ ion doped Ni L-Histidine Hydrochloride monohydrate crystals (Fe(III)-NiLHICL) are grown at room temperature using slow evaporation technique. The Fe(III) doped NiLHICL crystals are characterized by spectroscopic techniques such as X-Ray diffraction studies, Electron Paramagnetic Resonance(EPR), Optical Absorption and FTIR studies. Thermal stabilities were studied by TGA/DTA analysis. The powder diffraction patterns of prepared crystals have been recorded and lattice cell parameters are evaluated as $\mathrm{a}=1.5286, \mathrm{~b}=$ $0.8933, \mathrm{c}=0.6852 \mathrm{~nm}$. From EPR studies, g and hyperfine splitting parameters for $\mathrm{Fe}(\mathrm{III})$ ion in the host crystals are determined as $\mathrm{g}=2.0301$ indicating octahedral symmetry. Crystal field and inter-electronic parameters are evaluated from the optical absorption studies in addition to obtaining the confirmation for octahedral symmetry for the ions in the host lattice. The FT-IR spectrum exhibited characteristic vibrations of the groups present in the crystal indicating bond formation between the metal ion and the amino acid.
\end{abstract}

Keywords: Crystal field, FT-IR, Optical absorption spectrum, TGA,DTA, XRD.

\section{INTRODUCTION}

Doping is a well chosen and widely accepted technique for incorporating the required stability, electrical and optical properties in a bulk material. The technique has been explored extensively to modify the properties like photoluminescence, conductivity and crystal growth. Metal ion dopants, especially transition metal ion dopants modify the properties of a crystal for technological applications [1-4].

Amino acid crystals grown from aqueous solutions containing transition metal ions constitutes a solid state study to understand the role of transition metal ions in proteins. Many proteins contain metal ions and these metal ions perform a variety of specific functions associated with life processes. Transition metals such as $\mathrm{Fe}$, $\mathrm{Cu}$ and $\mathrm{Mn}$ are involved in many redox processes which require electron transfer and play an important role in the bio-functionality of proteins [5]. Amino acid L-Histidine is a component of proteins and is an enzyme active site. Structurally it possesses four potential coordination sites; the carboxyl group, the amino group and the pyridinic and pyrollic nitrogens from the imadazole residue [6]. The imadazole pyridine nitrogen is the strongest electron pair donor to the metal ions. Biologically L-Histidine is involved in a large number of biochemical processes and is now used in the treatment of anemia, allergies, rheumatoid arthritis, and other inflammatory reactions [7].

Potentiality of L-Histidine complexes was once again realised with the discovery of a ternary system of $\mathrm{Cu}$ (II) mixed ligand amino acid complex, L-Glutamine-Copper(II)-L-Histidine [8] for the treatment of Menkes disease, a lethal genetic disorder which can be treated by proper transportation of $\mathrm{Cu}(\mathrm{II})$ and for which a physiological path was discovered.

Winkler et al. presented a study of low-concentration Fe (III) doping in a crystalline amino acid, LAlanine by means of EPR and Raman scattering[9]. The paper did put forward an interesting observations on $\mathrm{Fe}(\mathrm{III})$ doping as though $\mathrm{Fe}$ is one of the most important transition metal ions in living organisms, Fe-doped amino acid crystals have not been studied and thus crystal structure revealing the position of the Fe impurities and their interactions with the molecular field of the crystal are not clear. The reasons, according to them are: Firstly, the crystal growth process is difficult to control for Fe dopants compared to other metal ions, $\mathrm{Fe}$ impurities are accepted in rather low concentrations and small concentrations of $\mathrm{Fe}$ and uneven small crystals create problems in the determination of impurity positions. Secondly, in the case of amino acids like L-Alanine, the large spin-lattice relaxations were observed in Fe-doped crystals which did not enable the EPR spectra of Fe (III) above $30 \mathrm{~K}$, an additional experimental difficulty. Thirdly, the super hyperfine interactions between the electronic spin of a given Fe impurity and the nuclear spins of its neighbours cannot be resolved. They concluded that $\mathrm{Fe}$ (III) occupied two in-equivalent sites of rhombic symmetry in the L-Alanine crystal. Continuing on these lines, we have grown and characterized Fe (III) doped Ni (II) L-Histidine hydrochloride monohydrate crystals. 


\section{EXPERIMENTAL DETAILS}

The crystals of Nickel L-Histidine Hydrochloride monohydrate here after called NiLHICL are grown by slow evaporation at room temperature from the aqueous, equimolar and equivolume solutions containing Nickel chloride hexahydrate $\left(\mathrm{NiCl}_{2} \cdot 6 \mathrm{H}_{2} \mathrm{O}\right)$ and L-Histidine hydrochloride monohydrate $\left(\mathrm{C}_{6} \mathrm{H}_{10} \mathrm{~N}_{3} \mathrm{O}_{2} \mathrm{Cl} . \mathrm{H}_{2} \mathrm{O}\right)$. By adding $0.01 \mathrm{~mol} \%$ of ferric chloride to the growth solution yields Fe (III)-NiLHICL crystals about fifteen days by slow evaporation. Powder XRD spectrum of the prepared crystals were recorded on PHILIPS Make PW1830 X-RAY Diffract meter, Polycrystalline EPR spectrum is recorded at room temperature (RT) on JEOL- JES-FA 200 EPR spectrometer, Crystals of $2 \mathrm{~mm}$ thickness were selected for optical absorption spectra recorded on JASCO V670 spectrophotometer. FT-IR spectra were recorded using KBr pellets on Thermo Nicolet 6700 FTIR spectrophotometer in the region $400-4000 \mathrm{~cm}-1$. The samples were tested using Q-switched Nd:Yag laser (1064nm, Quanta ray series) supplied by Spectra Physics, USA and Coherent Molectron powermeter, USA. Thermal studies were carried out using SII nano technology model TGA/DTA 6200 make Japan.

\section{RESULTS AND DISCUSSION}

\subsection{Powder XRD studies}

Powder X-ray diffraction for powder samples of Fe (III)-NiLHICL crystals revealed sharp, clean patterns indicative of highly crystalline phases. The powder XRD pattern of the prepared sample is shown in Fig.1. Lattice cell parameters are evaluated using Nakamoto Programme for Fe (III)-NiLHICL crystals as a = $1.5286 \mathrm{~nm}, \mathrm{~b}=0.8933 \mathrm{~nm}, \mathrm{c}=0.6852 \mathrm{~nm}$. These values agree well with the reported values of pure LHICL

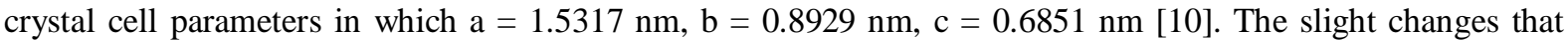
have occurred in the lattice parameters may be due to complexation or presence of doped metal ions in crystal lattice structure[11]. It is also an indication that presence of metal ions could not influence the cell parameters and the lattice structure to a greater extent.[12]. d-Spacings and $h k l$ values of Fe (III) doped NiLHICl crystal are given in Table1.The powder X-ray diffraction data for pure LHICL and Fe (III) doped NiLHICL crystals are given in the Table. 2

\subsection{EPR Studies}

The EPR spectrum of a poly crystalline sample revealed two intense resonance signals at $\mathrm{g}=4.143$ and $\mathrm{g}=2.030$ which are characteristic of $\mathrm{Fe}^{+3}$ ion. The spectrum is given in Fig 2 . The value nearly at $\mathrm{g} \approx 4.2 \pm 0.1$ is in general due to isolated $\mathrm{Fe}^{+3}$ ions located in sites of distorted octahedral symmetry, either rhombic or tetragonal from strong crystal field effects and $\mathrm{g} \approx 2$ was attributed to $\mathrm{Fe}^{+3}$ ion interactions by dipole- dipole interaction in a site of less distorted octahedral field [ 13-15]. The $\mathrm{g}$ values, $\mathrm{g}=4.143$ and $\mathrm{g}=2.030$ in low magnetic and high magnetic fields obtained for Fe(III) doped Ni LHICL crystal can also be interpreted on the same lines.

Fe (III) ions belong to $\mathrm{d}^{5}$ electronic configuration with ${ }^{6} \mathrm{~S}_{5 / 2}$ ground state in the free ion and the spins are almost free to orient themselves in external magnetic field with no spin-orbit interaction [16]. When $\mathrm{Fe}^{+3}$ ions are introduced in a crystal field environment, ${ }^{6} \mathrm{~S}$ ground state splits into three Kramer doublets. $| \pm 1 / 2>|$ $\pm 3 / 2>\mid \pm 5 / 2$ and resonance signal at $g=4.1$ results from the middle Kramers doublet $( \pm 3 / 2)$ [32]. Therefore, we can conclude that $\mathrm{g}$ values obtained correspond to distribution of iron ions in the host lattice in distorted octahedral symmetry.

Nickel (II) is a $\mathrm{d}^{8}$ ion and a non-Kramer ion. For a non-Kramer ion EPR activity is observable generally at low temperatures and the room temperature recordings did not show any EPR signals. [17-25]

\subsection{Optical absorption studies}

For a $\mathrm{d}^{5}$ configuration and with ${ }^{6} \mathrm{~s}$ as ground state, there are no spin-allowed transitions. The optical absorption spectrum of Fe(III) doped Ni LHICL is shown in Fig. 3 and is characteristic of $\mathrm{Fe}^{+3}$ in octahedral symmetry. The spectrum exhibits two weak bands at $580 \mathrm{~nm}$ and $725 \mathrm{~nm}$ which are characteristic of $\mathrm{Fe}^{3+}$ ions, assigned to the transitions corresponding to

$$
{ }^{6} \mathrm{~A}_{1 \mathrm{~g}}(\mathrm{~S}) \rightarrow{ }^{4} \mathrm{~T}_{1 \mathrm{~g}}(\mathrm{G}) \text { and }{ }^{6} \mathrm{~A}_{1(\mathrm{~g})}(\mathrm{S}) \rightarrow{ }^{4} \mathrm{~T}_{2 \mathrm{~g}}(\mathrm{D})
$$

The observed band positions are compared with those found in many $\mathrm{Fe}^{+3}$ doped systems, concluding that iron ions are present in a trivalent state with octahedral symmetry [26]. Crystal field and inter electronic repulsion parameters are evaluated as $\mathrm{Dq}=700 \mathrm{~cm}^{-1}, \mathrm{~B}=610 \mathrm{~cm}^{-1}$ and $\mathrm{C}=2360 \mathrm{~cm}^{-1}$. The value of inter electronic repulsion parameter $\mathrm{B}=610 \mathrm{~cm}^{-1}$ is far away from the free ion value $\left(1300 \mathrm{~cm}^{-1}\right)$ indicating a moderate covalent bonding [27].

The optical absorption spectrum also exhibited three characteristic bands for Ni (II) ion in the host lattice. Among the transition metal ions, $\mathrm{Ni}^{+2}$ ion exhibits strong absorption bands in the region from 200-1500 $\mathrm{nm}$ [28]. The three intense bands observed at $1110 \mathrm{~cm}^{-1}, 675 \mathrm{~cm}^{-1}$ and $422 \mathrm{~cm}^{-1}$ are characteristic of Ni(II) and are assigned to three spin allowed transitions corresponding to

$$
{ }^{3} \mathrm{~A}_{2 \mathrm{~g}}(\mathrm{~F}) \rightarrow{ }^{3} \mathrm{~T}_{2 \mathrm{~g}}(\mathrm{~F}),{ }^{3} \mathrm{~A}_{2 \mathrm{~g}}(\mathrm{~F}) \rightarrow{ }^{3} \mathrm{~T}_{1 \mathrm{~g}}(\mathrm{~F}) \text { and }{ }^{3} \mathrm{~A}_{2 \mathrm{~g}}(\mathrm{~F}) \rightarrow{ }^{3} \mathrm{~T}_{1(\mathrm{~g})} \mathrm{P}
$$


Based on these assignments energy matrices for $\mathrm{d}^{8}$ are solved. The values for crystal field and inter electronic repulsion parameters are evaluated as $\mathrm{Dq}=910 \mathrm{~cm}^{-1}$, $\mathrm{B}=775 \mathrm{~cm}^{-1}$ and $\mathrm{C}=2650 \mathrm{~cm}^{-1}$. The observed and calculated band head positions of Fe (III) doped Ni HICL crystals are listed in Table. 3

\subsection{FT-IR Studies}

FT-IR spectra are effectively used to identify the functional groups in the grown crystal. FT-IR spectrum of the Fe (III) doped Ni LHICL crystals is shown in Fig. 4. All the stretching and bending modes of FT-IR values of the Fe (III) doped Ni LHICL crystals are presented in Table. 4 The assignments for peaks/bands are given in accordance with the data in the literature [29-31]

The $\mathrm{NH}_{3}{ }^{+}$stretching and characteristic of hydrogen bonding region shows broad bands in the range $3500-2500 \mathrm{~cm}^{-1}$. The $\mathrm{N}-\mathrm{H}$ stretching vibration of the amino group in L-Histidine gives rise to an amide band between $3300 \mathrm{~cm}^{-1}$ and $3250 \mathrm{~cm}^{-1}$. The amide band is usually part of a Fermi resonance doublet with the second component absorbing weakly between 3100 and $3050 \mathrm{~cm}^{-1}$ [32]. The $\mathrm{CH}_{2}$ group of Histidine produces peaks at 2590 and $3000 \mathrm{~cm}^{-1}$ due to its symmetric and asymmetric stretching modes. The peak at $1568 \mathrm{~cm}^{-1}$ is attributed to the skeletal vibrations of Histidine ring. The obtained values are compared with the values of pure LHistidine [33] and doping of L-Histidine hydrochloride monohydrate crystal [34].

\subsection{Non linear optical (NLO) Studies}

The nonlinear optical conversion efficiency test was carried out for the grown crystals using the KurtzPerry powder technique [35]. KDP was used as reference material for the present measurement. The emission of green light confirmed generation of second harmonic radiation. SHG efficiency of Fe (III) doped Ni HICL is 0.9 times that of KDP. N.L.O parameters are shown in Table.5

\subsection{TGA/DTA Studies}

Thermogravimetric data provides information about decomposition pattern of materials in terms of weight loss and are limited to decomposition and oxidation reactions. Thermal studies were carried out in the temperature range $0-700{ }^{\circ} \mathrm{C}$ and thermogram recorded is shown in shown in Fig.5. A weight loss of $13.9 \%$ was recorded in the temperature range $0{ }^{\circ} \mathrm{C}-183.1^{\circ} \mathrm{C}$ indicated that the sample is thermodynamically stable up to a temperature of $183.1^{\circ} \mathrm{C}$. The peak at $183.1{ }^{\circ} \mathrm{C}$ was due to release of water molecules from crystal structure. The percentage weight loss decreased gradually up to $600^{\circ} \mathrm{C}$

DTA curve of the sample revealed no endothermic peak below $183.1^{\circ} \mathrm{C}$ suggesting its structural stability up to this temperature. This ensures the suitability of the material for possible applications in lasers where the crystal is required to withstand this temperature range. It is followed by two more peaks at $266.7^{\circ} \mathrm{C}$ and $569.5^{\circ} \mathrm{C}$ which are due to volatisation of the compound confirming a reasonable range of temperature for application of these materials .

\section{FIGURES AND TABLES}

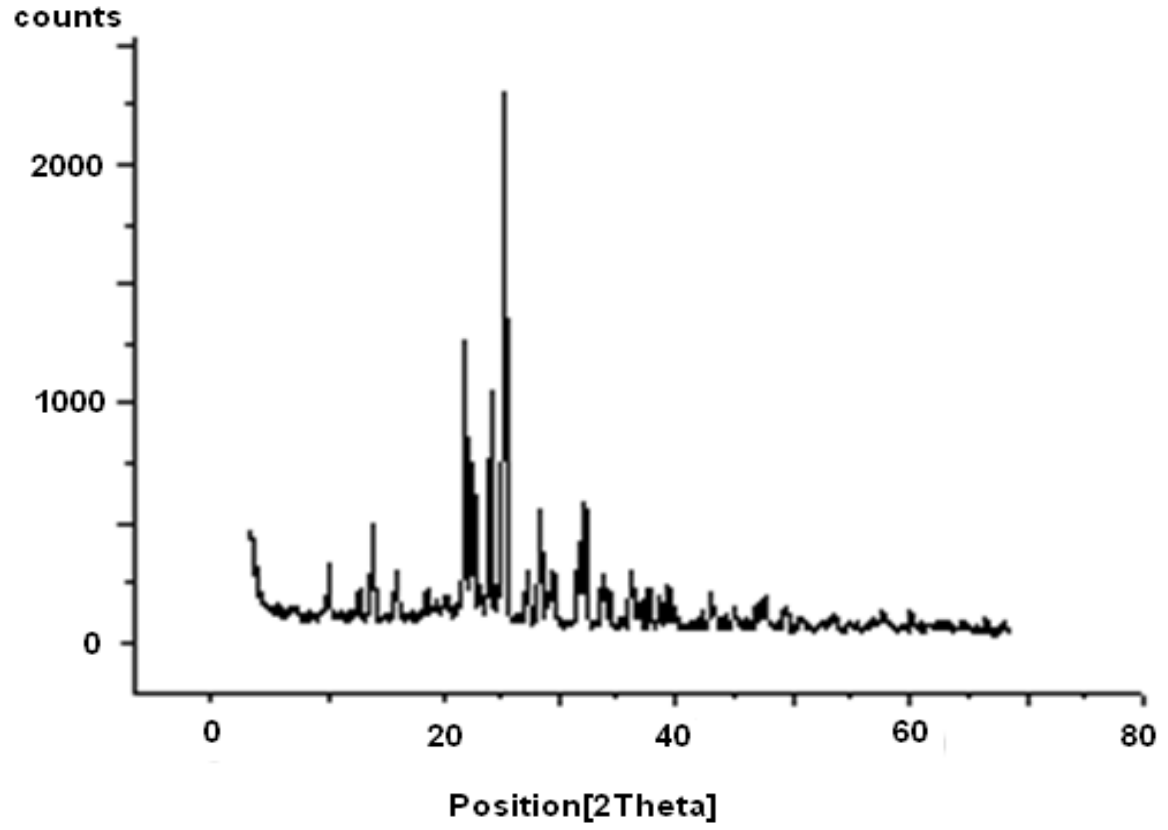

Fig.1 XRD spectrum of Fe (III) doped Ni LHICL. 


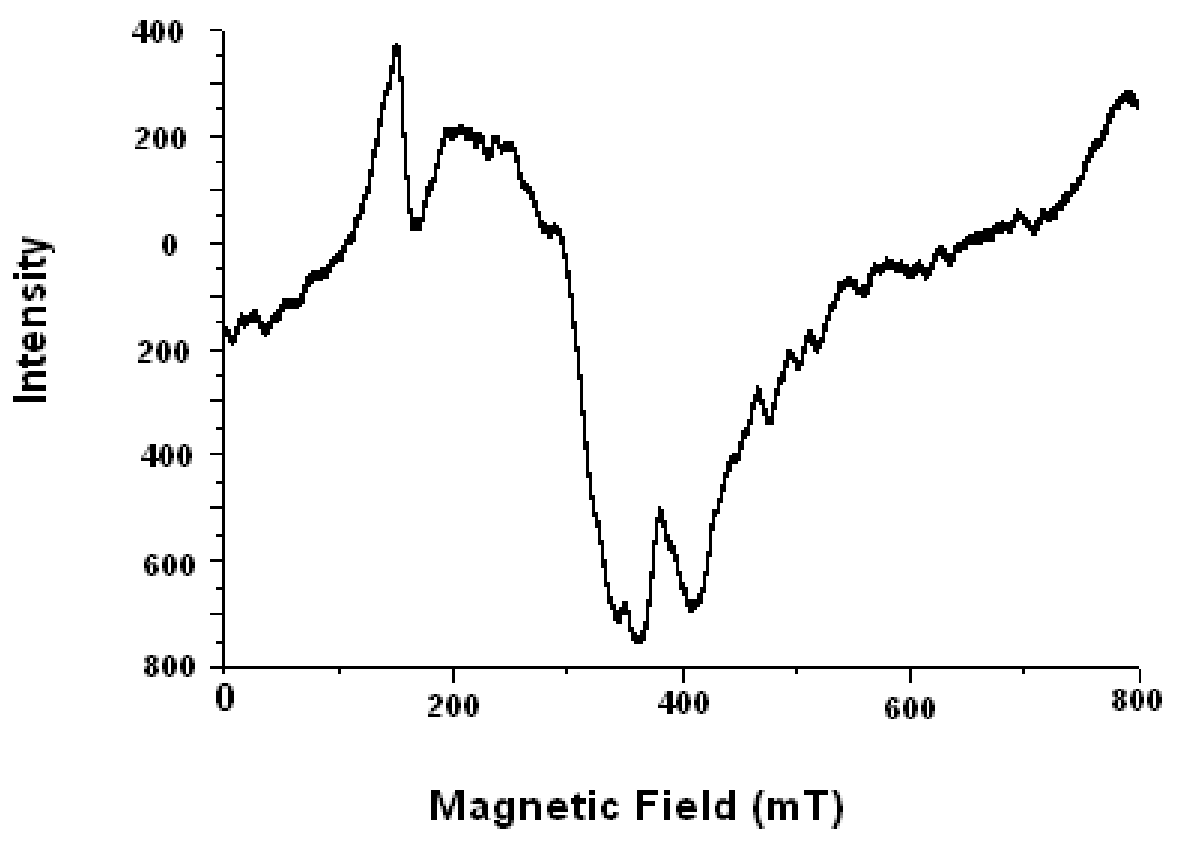

Fig.2 EPR spectrum of Fe(III) doped Ni LHICL

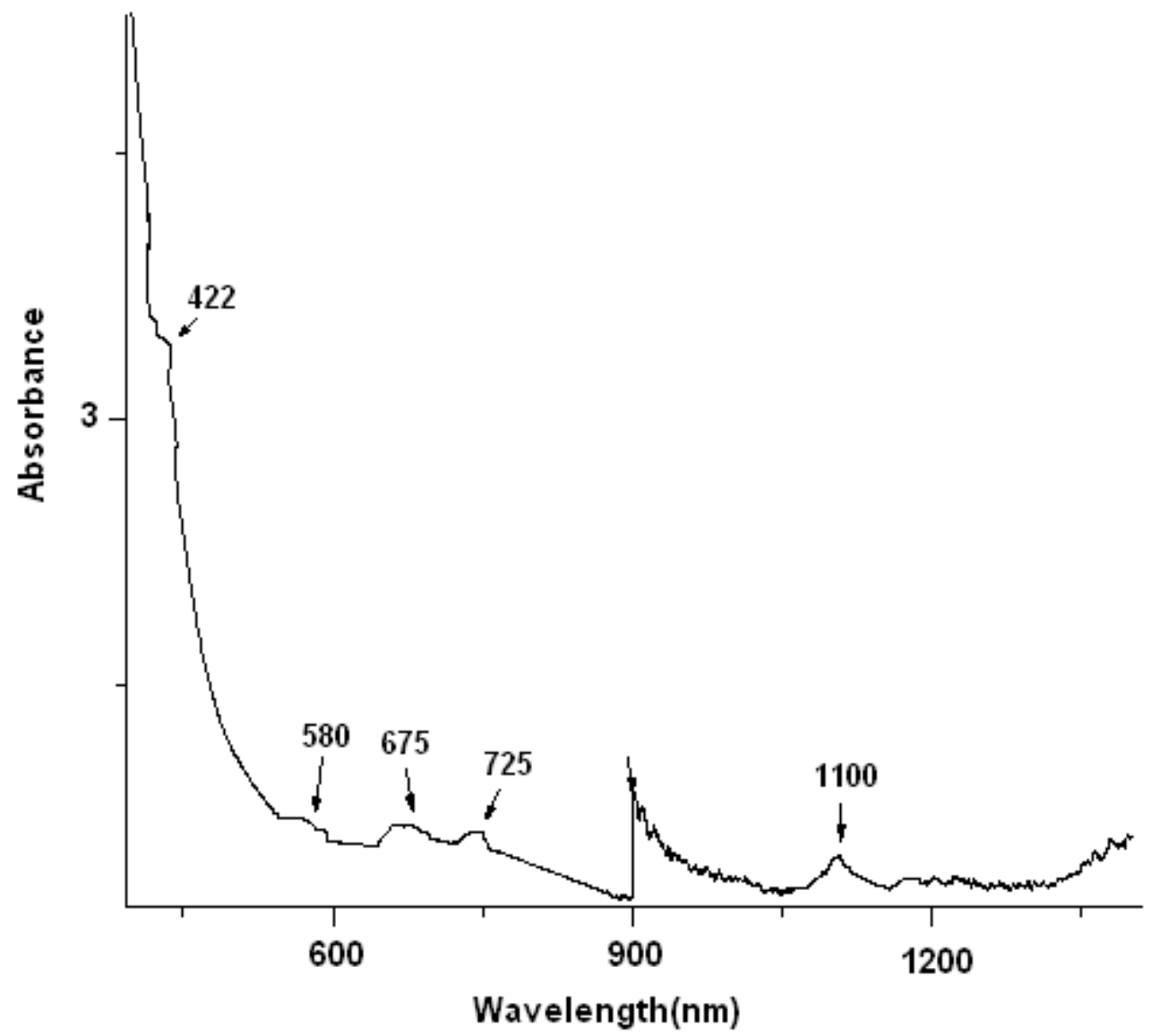

Fig.3 Optical absorption spectrum of Fe (III) doped Ni LHICL 


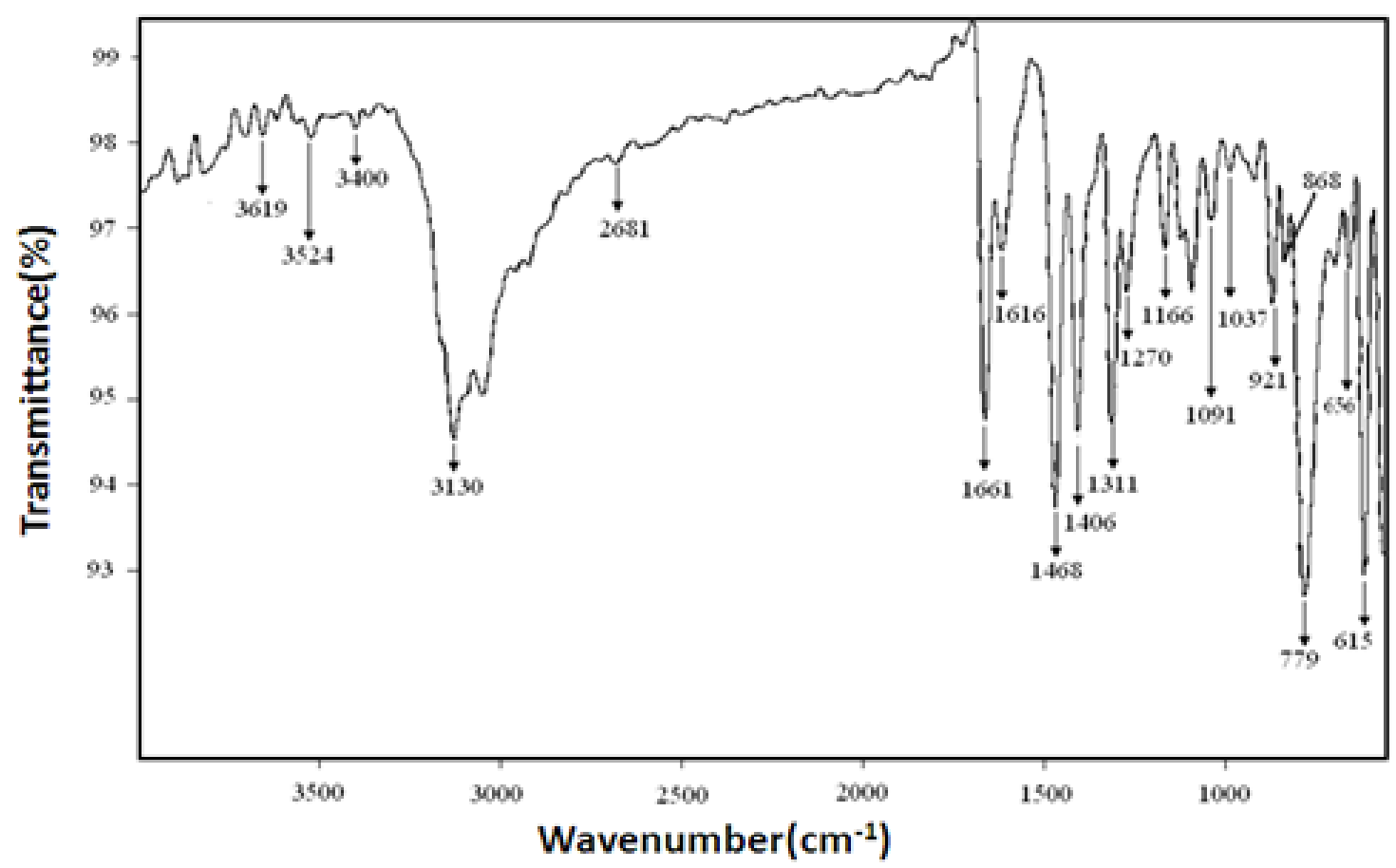

Fig.4 FT-IR spectrum of Fe(III) doped Ni LHICL

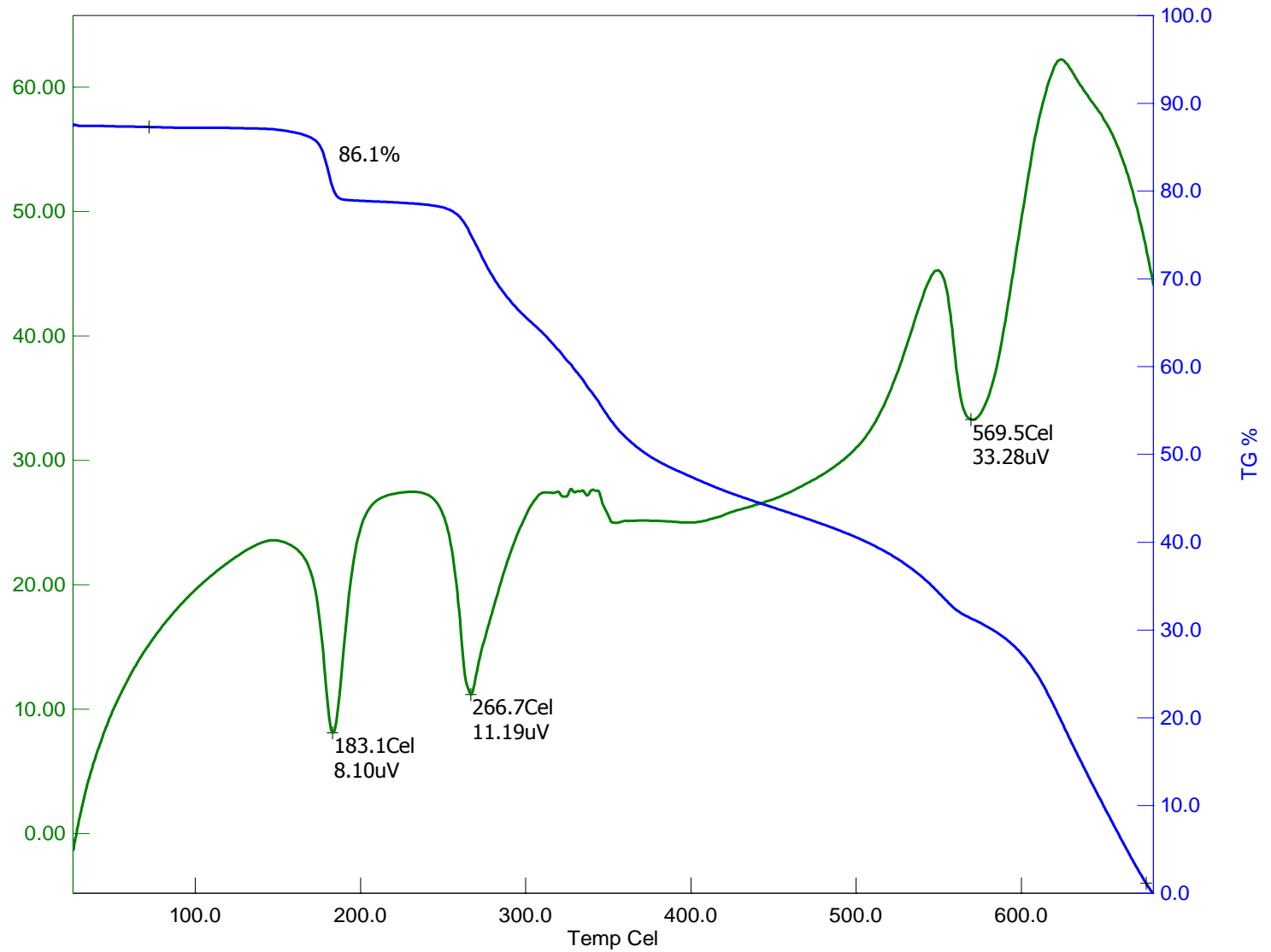


Growth, Characterization and NLO activity of Fe (III) doped Ni L-Histidine Hydrochloride

Table. 1 d-Spacings and $h k l$ values of Fe (III) doped Ni LHICL crystal.

\begin{tabular}{|c|c|c|c|c|c|c|}
\hline \multicolumn{2}{|c|}{ d - Spacing } & \multicolumn{3}{c|}{ Indices } & \multicolumn{2}{c|}{} \\
\hline Observed & Calculated & $\boldsymbol{h}$ & $\boldsymbol{K}$ & $\boldsymbol{l}$ & Observed & Calculated \\
\hline 6.18 & 6.25 & 1 & 1 & 0 & 14.36 & 14.36 \\
\hline 5.76 & 5.79 & 2 & 1 & 0 & 15.36 & 15.27 \\
\hline 5.06 & 5.10 & 2 & 0 & 1 & 17.49 & 17.36 \\
\hline 4.41 & 4.41 & 3 & 1 & 0 & 20.11 & 20.08 \\
\hline 3.79 & 3.81 & 4 & 0 & 0 & 23.42 & 23.29 \\
\hline 3.69 & 3.71 & 3 & 1 & 1 & 24.07 & 23.97 \\
\hline 3.60 & 3.62 & 1 & 2 & 1 & 24.69 & 24.51 \\
\hline 3.48 & 3.50 & 4 & 1 & 0 & 25.57 & 26.37 \\
\hline 3.32 & 3.33 & 4 & 0 & 1 & 26.78 & 26.71 \\
\hline 3.11 & 3.12 & 2 & 0 & 2 & 28.63 & 28.50 \\
\hline 2.99 & 3.01 & 3 & 2 & 1 & 29.76 & 29.64 \\
\hline 2.88 & 2.88 & 5 & 1 & 0 & 30.96 & 30.94 \\
\hline 2.70 & 2.70 & 3 & 1 & 2 & 33.09 & 33.03 \\
\hline 2.55 & 2.55 & 4 & 0 & 2 & 35.14 & 35.14 \\
\hline 2.51 & 2.51 & 5 & 2 & 0 & 35.63 & 35.62 \\
\hline 2.39 & 2.39 & 3 & 2 & 2 & 37.55 & 37.48 \\
\hline 2.33 & 2.34 & 4 & 3 & 0 & 38.45 & 38.38 \\
\hline 2.30 & 2.28 & 5 & 0 & 2 & 39.40 & 39.48 \\
\hline 2.25 & 2.24 & 0 & 3 & 2 & 40.02 & 40.13 \\
\hline 2.21 & 2.21 & 4 & 2 & 2 & 40.70 & 40.72 \\
\hline 2.07 & 2.07 & 7 & 0 & 1 & 43.55 & 43.51 \\
\hline 2.03 & 2.03 & 5 & 3 & 1 & 44.41 & 44.53 \\
\hline 1.98 & 1.99 & 6 & 1 & 2 & 45.61 & 45.52 \\
\hline 1.95 & 1.95 & 3 & 4 & 1 & 46.38 & 46.39 \\
\hline 1.88 & 1.88 & 7 & 2 & 1 & 48.28 & 48.29 \\
\hline 1.85 & 1.85 & 6 & 2 & 2 & 48.98 & 49.00 \\
\hline 1.79 & 1.79 & 1 & 3 & 3 & 50.74 & 50.69 \\
\hline 1.75 & 1.75 & 3 & 4 & 2 & 52.02 & 52.11 \\
\hline 1.66 & 1.66 & 8 & 0 & 2 & 54.97 & 55.03 \\
\hline 1.56 & 1.56 & 8 & 2 & 2 & 59.02 & 59.11 \\
\hline 1.06 & 1.50 & 6 & 4 & 2 & 61.55 & 61.54 \\
\hline & & & & & & \\
\hline
\end{tabular}

Table. 2 XRD data of pure LHICI and Fe(III) doped Ni LHICL crystals.

\begin{tabular}{|c|c|c|c|c|}
\hline Sample & $\begin{array}{c}\text { Cell } \\
\text { Parameters(nm) }\end{array}$ & Crystal System & Space Group & Volume/ $\mathbf{A}^{\mathbf{3}}$ \\
\hline & $\begin{array}{l}\mathrm{a}=1.5317 \\
\mathrm{~b}=0.8929 \\
\mathrm{c}=0.6852\end{array}$ & Orthorhombic & $\mathrm{P}_{21,21,21}$ & 933.71 \\
\hline Pure LHICL & $\begin{array}{l}\mathrm{a}=1.5286 \\
\mathrm{~b}=0.8923 \\
\mathrm{c}=0.6852\end{array}$ & Orthorhombic & $\mathrm{P}_{21,21,21}$ & 935.83 \\
\hline $\begin{array}{c}\text { Fe(III) doped Ni } \\
\text { LHICL }\end{array}$ & & & \\
\hline
\end{tabular}


Table. 3 Optical values of Fe (III) doped Ni LHICL crystal. Observed and calculated energies of various bands in the optical absorption spectrum of Fe (III) doped Ni LHICL.

\begin{tabular}{|c|c|c|c|}
\hline $\begin{array}{c}\text { Transitions From } \\
{ }_{6}^{6} \mathbf{A}_{1 \mathrm{~g}}(\mathbf{S})\end{array}$ & Wavelength (nm) & $\begin{array}{c}\text { Observed } \\
\text { Wavenumber }\left(\mathrm{cm}^{-1}\right)\end{array}$ & $\begin{array}{c}\text { Calculated } \\
\text { Wavenumber }\left(\mathrm{cm}^{-1}\right)\end{array}$ \\
\hline \multicolumn{4}{|l|}{$\underline{\text { For Fe(III) }}$} \\
\hline${ }^{6} \mathrm{~A}_{1 \mathrm{~g}}(\mathrm{~S}) \rightarrow{ }^{4} \mathrm{~T}_{1 \mathrm{~g}}(\mathrm{G})$ & 580 & 17236 & 17262 \\
\hline${ }^{6} \mathrm{~A}_{1 \mathrm{~g}}(\mathrm{~S}) \rightarrow{ }^{4} \mathrm{~T}_{2 \mathrm{~g}}(\mathrm{G})$ & 725 & 13789 & 13729 \\
\hline \multicolumn{2}{|c|}{$\mathrm{Dq}=700 \mathrm{~cm}^{-1}$} & \multicolumn{2}{|c|}{$[\alpha]=90 \mathrm{~cm}^{-1}$} \\
\hline \multicolumn{4}{|l|}{$\underline{\text { For } \mathrm{Ni}(\mathrm{II})}$} \\
\hline${ }^{3} \mathrm{~A}_{2 \mathrm{~g}}(\mathrm{~F}) \rightarrow{ }^{1} \mathrm{~T}_{1 \mathrm{~g}}(\mathrm{~F})$ & 422 & 23690 & 23700 \\
\hline${ }^{3} \mathrm{~A}_{2 \mathrm{~g}}(\mathrm{~F}) \rightarrow{ }^{3} \mathrm{~T}_{1 \mathrm{~g}}(\mathrm{P})$ & 675 & 14765 & 14690 \\
\hline${ }^{3} \mathrm{~A}_{2 \mathrm{~g}}(\mathrm{~F}) \rightarrow{ }^{3} \mathrm{~T}_{2 \mathrm{~g}}(\mathrm{~F})$ & 1100 & 9088 & 9100 \\
\hline \multicolumn{2}{|c|}{$\mathrm{Dq}=910 \mathrm{~cm}^{-1}$} & \multicolumn{2}{|c|}{$\mathrm{C}=2650 \mathrm{~cm}^{-1}$} \\
\hline
\end{tabular}

Table. 4 Observed Vibrational modes of Fe(III) doped Ni LHICL.

\begin{tabular}{|c|l|}
\hline Wavenumber $\left(\mathbf{c m}^{-1}\right)$ & Assignments \\
\hline 3403 & O-H stretching of water \\
\hline 2997 & N-H Symmetrical stretching \\
\hline 2616 & C-H Symmetrical stretching \\
\hline 1635 & $\mathrm{C}=\mathrm{O}$ stretching \\
\hline 1616 & Asymmetric bend of $\mathrm{NH}_{3}{ }^{+}$and $\mathrm{C}=\mathrm{N}$ stretching \\
\hline 1568 & Asymmetric mode of $-\mathrm{COO}^{-}$and $\mathrm{C}=\mathrm{C}$ stretching \\
\hline 1470 & Symmetric bend of $\mathrm{NH}_{3}$ \\
\hline 1406 & Symmetric mode of $-\mathrm{COO}^{-}$and C-N stretching \\
\hline 1070 & C-O stretching of carboxylic group \\
\hline 863 & C-H out of plane bending \\
\hline
\end{tabular}

Table .5 NLO test parameters of Fe(III) doped Ni LHICL.

\begin{tabular}{|l|l|}
\hline Input Wavelength $=1064 \mathrm{~nm}$ & Input power $=0.68 \mathrm{~J}$ \\
\hline $\mathrm{KDP}=8.8 \mathrm{~mJ}$ & $\mathrm{Fe}(\mathrm{III})$ doped $\mathrm{NiHICL}=6.8 \mathrm{~mJ}$ \\
\hline
\end{tabular}

\section{CONCLUSIONS}

Fe (III) doped Ni LHICL Crystals were grown at room temperature and were characterized by using Powder XRD, EPR, Optical absorption, FT-IR and TGA/DTA studies. The slight deviation of evaluated cell parameters from pure L-Histidine hydrochloride monohydrate crystals may be due to complexation and the incorporation of transition metal ions in to the host lattice. Evaluation of crystal field and Racah parameters from the optical absorption spectra confirms the coordination of Fe (III) ions in an octahedral site symmetry in the Ni LHICL host lattice. EPR spectral studies, through spin-Hamiltonian and hyperfine splitting parameters suggested distorted octahedral sites for Fe (III) ions in the host lattice. Correlated EPR and optical parameters suggested a partial covalence between Fe (III) ions and the host lattice. Fe(III)-Ni LHICL crystals showed different characteristic vibrational bands related to stretching modes of $\mathrm{NH}_{3}{ }^{+}, \mathrm{COO}^{-}$groups, and in plane $\mathrm{C}-\mathrm{H}$ deformation of imidazole ring confirming the presence of an amino acid. NLO studies indicated that $\mathrm{Fe}$ (III) 
doped Ni LHICL have second harmonic generation efficiency which was 0.9 times to that of KDP crystals and thermal studies indicated the suitability of the crystals for applications at high temperatures.

\section{ACKNOWLEDGMENTS}

The author YS is thankful to the University Grants Commission, Government of India, New Delhi for sanctioning the Major Research Project (F.37-7/2/2009 (SR)) to carry out the present research.

\section{REFERENCES}

[1] Hannay and C.F.Smyth, J.Am.Chem.Sot. 68, 1976,171.

[2] S.N.Rao, Y.P.Reddy and P.Sambasiva Rao, Solidi State Communications 82, 1992, 419.

[3] DAH Fenningham, RB. Hammond, LaiX and K J Roberts, Che matter, 7 1995, 1690.

[4] Lai X.Roberts K J Bedzyk J M.Lyman P F CardosoLp and JM Sasaki Chem matter.17,1985, 4053

[5] Wen-Chen Zheng, Phys.Status Solidi, B, 205, 1998, 627.

[6] Izhar Hussain,Margaret Goodgame, Jour.Chem.Soc.Pak. vol.14, 1, 1992 11-14.

[7] H. O. Marcy, M. J. Rosker, L. F. Warren et al., Optics Letters, 20, 3, 1995, 252-254,

[8] P. Zerrouk, N. ; MartensT.; Charlot, M.F.; Girerd, J. J.; Chaumeil, J. C.;Tomas, A.Journal Of Trace \& Microprobe Techniques. 21, 4, 2003.729-741.

[9] E. Winkler, P. Etchegoin, A. Fainstein, and C. Fainstein Phys. Rev. B 61, 2000, 1575-85

[10] J. Donohue, L.R. Lavine, J.S. Rollett, Acta Crystallogr. 9, 1956, 655.

[11] CMRRem'edios, W Paraguassu, J A Lima Jr, P T C Freire,J Mendes-Filho, FEAMelo, A S de Menezes, A O dos Santos,L P Cardoso and M A R Miranda. J. Phys.: Condens. Matter 20, 2008, 275209 (6pp)

[12] P. Praveen Kumar, V. Manivannan, S. Tamilselvan, S. Senthil, Victor Antony Raj, P. Sagayaraj, J. Madhavan Optics Communications 281, 2008, 2989-2995

[13] I. Ardelean, M. Peteanu, S. Filip, V. Simon, G. Gyory, Solid State Commun. 102, 1997.341

[14] C. Hirayama, J.G. Castle Jr., M. Kuriyama, Phys.Chem. Glasses 9, 1968,109.

[15] D.Loveridge, S.Parke, Phys. Chem. Glasses 12, 1971, 90

[16] J. Kliava, Phys. Status Solidi B 134, 1986. 411

[17] Sczaniecki P B and Lesiak J. Magn. Reson. 46, 1982, 185

[18] Sano W, Domiciano J B and Ochi J A Phys. Rev. 50, 1994, 5

[19] Kadish K M, Sazou D, Maiya G B, Han D C, Sasiabi A, Ferhat M and Guilard R Inorg. Chem. 28, 1989,2542

[20] Bencini, Alessandro, Farbrizzi, Luigi, Poggi and Antonio Inorg. Chem. 20, 1981, 2544

[21] Bencini A, Gatteschi D and Sacconi L Inorg. Chem. 17, 2768

[22] Rubins R S and Yung Y H J. Chem. Phys. 75, 1981, 4285

[23] Rubins R S and Haghighatojou T J. Phys. Chem. Solids 43, 1982, 491

[24] Zhao M G, Du M L and Sen G Y J. Phys. Chem. Solid State Phys. 33,1987, 5557

[25] Shrivastava K N, Rubenacker N, Hutton S L, Drumheller J E and Rubins R S J.Chem. Phys. 88, 1988,634

[26] R.P. Sreekanth chakradhar, G.Sivaramaih, J. Lakshmana Rao, N.O. Gopal Spectro chemical Acta PartA Molecular and Bio molecular Spectro scopy, 62, 1, 2005, 51-57

[27] B.N.Figgis. Introduction to Ligand Fields, Weily Eastern, NewDelhi1, p.23.

[28] R.Ramakumar and B.C.Venkata Reddy Acta physica Polonica A.Vol 871995.

[29] Nagamoto,K IR Spectra of Inorganic and Co ordination compound ( $^{\text {nd }}$ edition)Wiley\& Sons,NewYork,1978.

[30] Smith, B Infrared Spectral Interpretation: a Systematic Approach, CRC press, Washington, DC1999.

[31] Kalsi, P.S Spectroscopy of Organic Compounds, New Age International Ltd, New Delhi ,2002.

[32] R.Silverstein, G.C.Bassler, T.C.Morrill, spectroscopic identification of organic compounds, John Wiley \& Sons Singapore. 1981

[33] J.Madhavan, S.Aruna, P.C.Thomas, M.Vimalan, S.A.Rajasekar, P.Sagayaraj, Crystal Research Technology 42, 2007, 59.

[34] D.Syamala, V.Rajendran, R.K.Natarajan and S.MoorthyBabu, Journal of Crystal Growth and Design 7, 2007,1695

[35] K. Kurtz, T.T. Perry, J. Appl. Phys. 39, 1968, 3798. 